\title{
Approximate Chernoff Fusion of Gaussian Mixtures for Ballistic Target Tracking in the Re-entry Phase
}

Kelin Lu ${ }^{\mathrm{a}, *}$, Rui Zhou ${ }^{\mathrm{a}}$, Jie Zhang ${ }^{\mathrm{b}}$

\begin{abstract}
A track-to-track fusion method to combine local estimates modelled with Gaussian mixture model is proposed for tracking a re-entry ballistic vehicle. An arbitrary power of a Gaussian mixture distribution is approximated with Gaussian mixture model using first order expansion approximation, which leads to an analytical fusion equation for approximate Chernoff fusion. In the end, we verify the effectiveness of the proposed fusion algorithm with a series of Monte Carlo simulations.
\end{abstract}

Keywords: ballistic target tracking, Gaussian mixtures fusion, track-to-track fusion

\begin{tabular}{llll}
\hline Nomenclature & & \\
IMM & Interacting Multiple Model & $q$ & Process Noise Intensity \\
PDF & Probability Density Function & $T$ & Sampling Period \\
T2TF & Track-to-track Fusion & $F, G$ & Proper Dimension Matrices \\
KLD & Kullback-Leibler Divergence & $f(\cdot)$ & Aerodynamic Drag Acceleration \\
RMSE & Root Mean Squared Error & $g$ & Gravity Acceleration \\
CC & Convex Combination & $\beta$ & Ballistic Coefficient \\
CI & Covariance Intersection & $\rho(\cdot)$ & Function of Air Density \\
EI & Ellipsoidal Intersection & $y_{p}$ & Target Altitude \\
CFG & Configuration & $z$ & Measurement Vector \\
ACF & Approximate Chernoff Fusion & $h(\cdot)$ & Measurement Function \\
GM & Gaussian Mixtures & $v$ & Measurement Noise \\
$x$ & Target State Vector & $r$ & Range Measurement \\
$\hat{x}$ & Estimation Mean & $\theta$ & Bearing Angle \\
$P$ & Estimation Covariance & & Measurement \\
$Q$ & Covariance of Process Noise & & \\
\hline
\end{tabular}




\section{Introduction}

The problem of re-entry ballistic vehicle tracking across the atmosphere $[1,2]$ has recently attracted considerable attention because of its important significance in successful interception of re-entry targets for safety $[3,4]$. Technically speaking, the problem is a typical recursive nonlinear state estimation problem, where the nonlinearity stems from both the target dynamics [5] and measurement functions [5]. Several nonlinear estimation methods have been used for ballistic target tracking, including extended Kalman filter [6], unscented Kalman filter [7], particle filter [8] and ensemble Kalman filter [2]. One disadvantage of these methods is that they assume the ballistic coefficient, which is one of key parameters for target dynamics, is known with sufficient accuracy. However, the ballistic coefficient can only be known crudely or even unknown in practice [9]. In such a case, one idea is to consider that the target dynamics updates according to one of a bank of models, as the models can differ in values of ballistic coefficient. This assumption leads to the adoption of multiple model $[10,11]$ estimation methods, which incorporates multiple local filters to overcome the problem of estimation with unknown system parameters. As a result, the output of the local multiple model filters is in terms of Gaussian mixture model [12], which is obtained by weighted sum of estimates from each local filter.

Beyond one single sensor level, one could further increase the estimation accuracy of target state by means of track-to-track fusion (T2TF) techniques using local estimation results from multiple sensors. The basic T2TF system [13-15] includes a fusion center and at least two sensors, in which every sensor generates local estimates with its own measurements. The objective of the fusion center is to combine the local estimation tracks to generate a global track with improved tracking accuracy. While there has been a great deal of research work with regards to T2TF problem in literature, most of existed solutions [16-19] have been specifically focused on Gaussian distribution-based system, which means that these fusion algorithms might not be applicable to the estimation results from the multiple model filters. Therefore, it is necessary to develop novel fusion algorithm to directly fuse tracks in terms of Gaussian mixture model. To this end, we first approximate an arbitrary power of a
Gaussian mixture distribution with Gaussian mixture model using first order expansion approximation. After that, we derive an analytical fusion equation for Gaussian mixtures (GM) based on the Chernoff fusion rule. Simulation result shows that the proposed fusion algorithm increases tracking accuracy significantly compared to existed solutions.

\section{Problem formulation}

The sensor fusion framework for tracking a reentry ballistic vehicle manly consists of two components. The first component is responsible for local filtering at the single sensor level, it models target dynamics, measurement equation, and gives a local estimate in terms of a Gaussian mixture model. The second component enables fusion of local estimates from the sensors. The diagram shown in Fig. 1 presents a graphical representation of the $\mathrm{T} 2 \mathrm{TF}$ architecture as proposed in this paper.

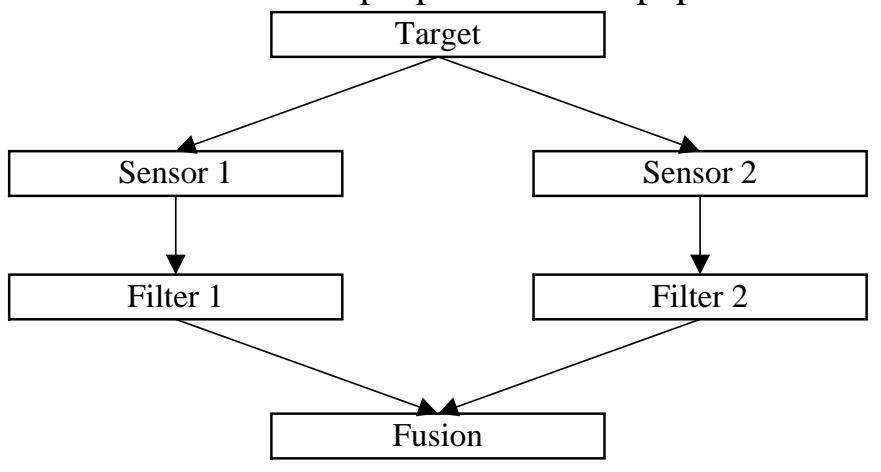

Fig. 1 Track-to-track fusion (T2TF) architecture

\subsection{Target dynamics}

In this work, the dynamics model of a re-entry ballistic vehicle is considered under the following assumptions [20]: the forces acting on the vehicle consists of the aerodynamic drag and Earth's gravity. The effects of Coriolis acceleration, centrifugal acceleration, lift, wind force and spinning motion are neglected due to their minor impact on the vehicle trajectory.

Therefore, the dynamics model of a re-entry ballistic vehicle is given in a Cartesian coordinate system as follows $[4,21]$

$$
x_{k+1}=\Psi\left(x_{k}\right)+G\left[\begin{array}{c}
0 \\
-g
\end{array}\right]+w_{k+1}
$$

where $x_{k}=\left[x_{p, k}, x_{v, k}, y_{p, k}, y_{v, k}\right]^{T}$ is defined as the target state vector at time step $k, x_{p}$ and $y_{p}$ represent positions, $x_{v}$ and $y_{v}$ represent velocities in $\mathrm{X}, \mathrm{Y}$ axis 
respectively. The process noise $w_{k}$ is modelled as zero mean white noise, namely

$$
w_{k} \square N(0, Q)
$$

And the covariance $Q$ of the process noise is given as

$$
Q=q\left[\begin{array}{cc}
\Theta & 0 \\
0 & \Theta
\end{array}\right] \text { with } \Theta=\left[\begin{array}{cc}
T^{3} / 3 & T^{2} / 2 \\
T^{2} / 2 & T
\end{array}\right]
$$

where $q$ represents noise intensity. $\Psi(\cdot)$ in Eq. (1) is a nonlinear function given as

$$
\Psi\left(x_{k}\right)=F x_{k}+G f\left(x_{k}\right)
$$

with

$$
F=\left[\begin{array}{llll}
1 & T & 0 & 0 \\
0 & 1 & 0 & 0 \\
0 & 0 & 1 & T \\
0 & 0 & 0 & 1
\end{array}\right] \quad G=\left[\begin{array}{cc}
T^{2} / 2 & 0 \\
T & 0 \\
0 & T^{2} / 2 \\
0 & T
\end{array}\right]
$$

where $f(\cdot)$ represents the aerodynamic drag

$$
f\left(x_{k}\right)=-\frac{g \rho\left(y_{p, k}\right) \sqrt{x_{v, k}^{2}+y_{v, k}^{2}}\left[\begin{array}{l}
x_{v, k} \\
y_{v, k}
\end{array}\right]}{2 \beta}
$$

$g$ is gravity acceleration, $\beta$ represents ballistic coefficient. The air density $\rho(\cdot)$ decays with target altitude $y_{p, k}$ in exponential manner [22] as

$$
\rho\left(y_{p, k}\right)=c_{1} e^{-c_{2} y_{p, k}}
$$

\subsection{Measurement equation}

The measurements observed by the sensors include ranges and bearing angles. Suppose that there are two ground-radar sensors, $S_{1}$ and $S_{2}$ in the system, and the sensors are located at $\left(x_{R}^{S_{l}}, y_{R}^{S_{l}}\right)$ for $l=1,2$, respectively. Then the measurement equation is written as

$$
z_{k}^{S_{l}}=h\left(x_{k}\right)+v_{k}^{S_{l}} \quad \text { for } \quad l=1,2
$$

where the measurement vector $z_{k}^{S_{l}}=\left[r_{k}^{S_{l}}, \theta_{k}^{S_{l}}\right]^{T}$ consists of range $r_{k}^{S_{l}}$ and bearing $\theta_{k}$ with respect to sensor $S_{l}$. The nonlinear measurement equations can be further formulated as

$$
\begin{aligned}
& r_{k}^{S_{l}}=\sqrt{\left(x_{p, k}^{S_{l}}-x_{R}^{S_{l}}\right)^{2}+\left(y_{p, k}^{S_{l}}-y_{R}^{S_{l}}\right)^{2}}+v_{r, k}^{S_{l}} \\
& \theta_{k}^{S_{l}}=\tan ^{-1}\left(\frac{y_{p, k}^{S_{l}}-y_{R}^{S_{l}}}{x_{p, k}^{S_{l}}-x_{R}^{S_{l}}}\right)+v_{\theta, k}^{S_{l}}
\end{aligned}
$$

where the measurement noises are white Gaussian, namely

$$
\begin{gathered}
v_{r, k}^{S_{l}} \square N\left(0, R_{r}^{S_{l}}\right) \\
v_{\theta, k}^{S_{l}} \square N\left(0, R_{\theta}^{S_{l}}\right)
\end{gathered}
$$

\subsection{IMM filter}

In this work, it is considered that the real ballistic coefficient $\beta$ is not available to the ground-radar sensors. Therefore we use the interacting multiple model (IMM) [23] filter to overcome the model uncertainty problem caused by unknown ballistic coefficient. In the IMM filter it is assumed that the target dynamics obeys one of a set of models (for example, each model is constructed with a specific value of ballistic coefficient). In this regard, the output of IMM filter is in terms of Gaussian mixture model by merging each model-based state estimate [24]. A block diagram for one-step recursion of IMM filter with two models $\left(r^{S_{l}}=2\right)$ is shown in Fig.

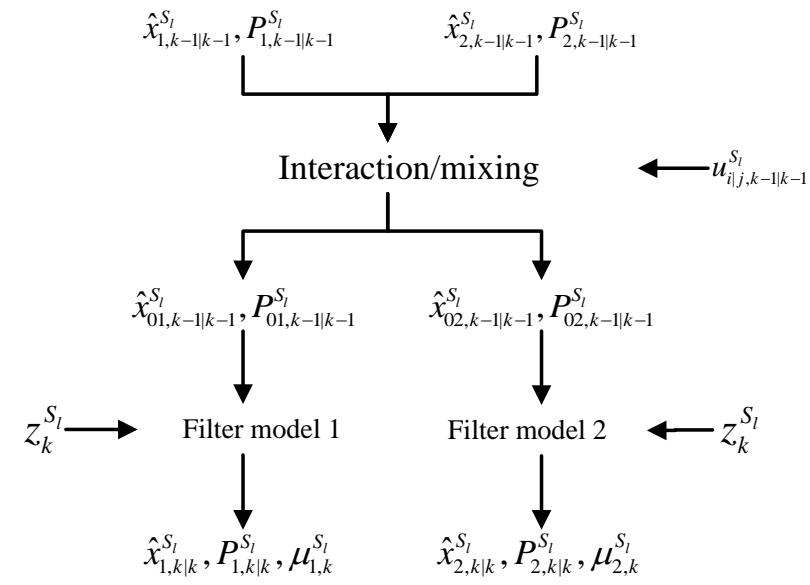

Fig. 2 Structure of the IMM filter for sensor $S_{l}$ (two filter models)

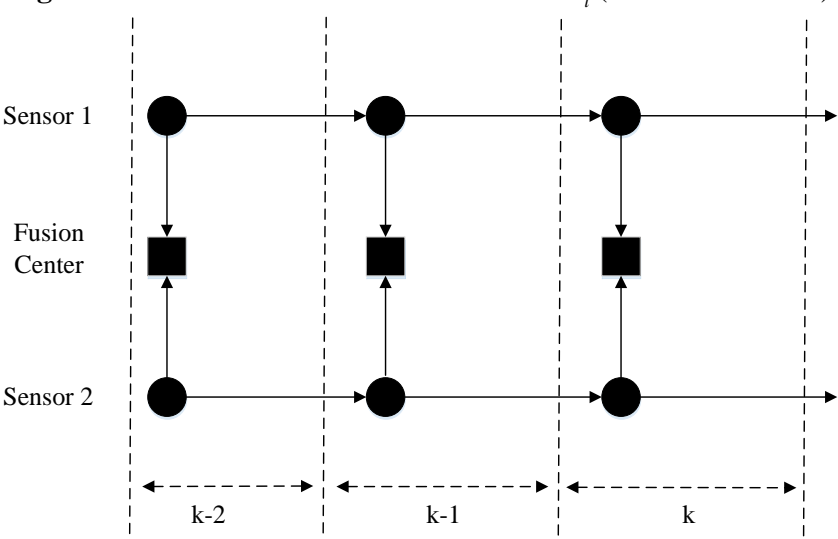

Fig. 3 Fusion process (full-rate communication)

\subsection{Fusion process}

In this paper, we consider that the local sensors run IMM filter for target tracking, thus the output of 
each local sensor is in terms of Gaussian mixture model. Consequently, the T2TF problem to solve here demands fusion of Gaussian mixture PDFs. As shown in Fig. 3, the fusion process includes two ground-radar sensors $S_{1}$ and $S_{2}$ with their local observations and estimations updated synchronously and periodically. The objective of the fusion center is to obtain a global estimate with improved tracking accuracy.

\section{Gaussian mixtures fusion}

\subsection{Optimal Bayesian fusion and suboptimal Chernoff fusion}

Consider a system with two sensors $S_{1}, S_{2}$ and a fusion center (FC). As shown in Fig. 3, each sensor transmits its up-to-date estimate to the FC with a synchronous communication interval for track fusion. The optimal fusion process, as described in Ref. [25], follows from the Bayes rule as

$$
p^{f}(x)=\frac{1}{c} \frac{p^{S_{1}}(x) p^{S_{2}}(x)}{p^{c}(x)}
$$

where $p^{S_{1}}(x)$ and $p^{S_{2}}(x)$ represent local estimates, $p^{c}(x)$ is the duplicate information to be removed [26]. Although the fusion equation (11) is straightforward [27], the accurate identification of $p^{c}(x)$ is complex and difficult in practice [13]. The major difficulty lies in the requirement to obtain the exact correlation between local estimates resulting from previous fusion events [26]. It becomes further difficult to fuse local estimation tracks in terms of Gaussian mixture model. To overcome the correlation problem, one practical solution is to use the Chernoff fusion rule [28] to obtain a suboptimal fused result with no need to consider the dependency between local estimates. The Chernoff fusion rule is formulated as

$$
p^{f}(x)=\frac{1}{c}\left(p^{S_{1}}(x)\right)^{w}\left(p^{S_{2}}(x)\right)^{1-w}
$$

where the parameter $w \in[0,1]$ is determined by minimizing some chosen criteria. It can be observed that Chernoff fusion is indeed a convex combination of local estimates in a log-linear manner [28]. Theoretically, Eq. (12) can be used to fuse arbitrary two probability density functions. However, an analytical-form solution only exists for Gaussian distribution case [29].

\subsection{Approximate Chernoff fusion for Gaussian mixture model}

In this section, we develop an approximate Chernoff fusion method to analytically fuse estimates in terms of Gaussian mixture model. To this end, we approximate an arbitrary power of a Gaussian mixture distribution with Gaussian mixture model using first order expansion approximation. Such an approximation can lead to a fused result with closed form expressions.

In the following, let us consider two Gaussian mixtures, $p^{S_{l}}(x)=\sum_{i=1}^{r^{S_{l}}} \mu_{i}^{S_{l}} N\left(x ; \hat{x}_{i}^{S_{l}}, P_{i}^{S_{l}}\right)$ for $l=1,2$ to be fused with fusion equation (12), then the fused estimate can be written as

$$
\begin{aligned}
p^{f}(x) & =\frac{1}{c}\left(\sum_{i=1}^{r^{S_{1}}} \mu_{i}^{S_{1}} N\left(x ; \hat{x}_{i}^{S_{1}}, P_{i}^{S_{1}}\right)\right)^{w} \\
& \cdot\left(\sum_{i=1}^{r_{2}} \mu_{i}^{S_{2}} N\left(x ; \hat{x}_{i}^{S_{2}}, P_{i}^{S_{2}}\right)\right)^{1-w}
\end{aligned}
$$

First, to calculate the power of Gaussian mixtures we use its first order expansion approximation as

$$
\begin{aligned}
\left(p^{S_{1}}(x)\right)^{w} & \approx \frac{\sum_{i=1}^{N^{S_{1}}}\left(\mu_{i}^{S_{1}}\right)^{w} N\left(x ; \hat{x}_{i}^{S_{1}}, \frac{P_{i}^{S_{1}}}{w}\right)}{C^{S_{1}}} \\
\left(p^{S_{2}}(x)\right)^{1-w} & \approx \frac{\sum_{j=1}^{N^{S_{2}}}\left(\mu_{j}^{S_{2}}\right)^{1-w} N\left(x ; \hat{x}_{j}^{S_{2}}, \frac{P_{j}^{S_{2}}}{1-w}\right)}{C^{S_{2}}}
\end{aligned}
$$

where $C^{S_{1}}=\sum_{i=1}^{N^{S_{1}}}\left(\mu_{i}^{S_{1}}\right)^{w}$ and $C^{S_{2}}=\sum_{j=1}^{N^{S_{2}}}\left(\mu_{j}^{S_{2}}\right)^{1-w}$ are normalization constants.

Substitute Eq. (14) into Eq. (13), it yields

$$
\begin{aligned}
& p^{f}(x) \\
= & \frac{1}{C^{f}} \sum_{i=1}^{N^{S_{1}}} \sum_{j=1}^{N^{S_{2}}}\left(\mu_{i}^{S_{1}}\right)^{w}\left(\mu_{j}^{S_{2}}\right)^{1-w} \\
& \cdot N\left(x ; \hat{x}_{i}^{S_{1}}, \frac{P_{i}^{S_{1}}}{w}\right) N\left(x ; \hat{x}_{j}^{S_{2}}, \frac{P_{j}^{S_{2}}}{1-w}\right) \\
= & \frac{1}{C^{f}} \sum_{i=1}^{N^{S_{1}}} \sum_{j=1}^{N^{S_{2}}}\left(\mu_{i}^{S_{1}}\right)^{w}\left(\mu_{j}^{S_{2}}\right)^{1-w} c_{i j} N\left(x ; \hat{x}_{i j}, P_{i j}\right)
\end{aligned}
$$

in which $C^{f}$ represents normalization constant, and 


$$
\begin{aligned}
P_{i j}^{-1} & =w\left(P_{i}^{S_{1}}\right)^{-1}+(1-w)\left(P_{j}^{S_{2}}\right)^{-1} \\
P_{i j}^{-1} \hat{x}_{i j} & =w\left(P_{i}^{S_{1}}\right)^{-1} \hat{x}_{i}^{S_{1}}+(1-w)\left(P_{j}^{S_{2}}\right)^{-1} \hat{x}_{j}^{S_{2}} \\
c_{i j} & =\exp \left(\zeta_{i}^{S_{1}}+\zeta_{j}^{S_{2}}-\zeta_{i j}\right)
\end{aligned}
$$

with

$$
\begin{aligned}
\zeta_{i}^{S_{1}} & =-\frac{1}{2}\left(d \log 2 \pi-\log \left|w\left(P_{i}^{S_{1}}\right)^{-1}\right|\right. \\
& \left.+w\left(\hat{x}_{i}^{S_{1}}\right)^{T}\left(P_{i}^{S_{1}}\right)^{-T} \hat{x}_{i}^{S_{1}}\right) \\
\zeta_{j}^{S_{2}}= & -\frac{1}{2}\left(d \log 2 \pi-\log \left|(1-w)\left(P_{j}^{S_{2}}\right)^{-1}\right|\right. \\
& \left.+(1-w)\left(\hat{x}_{j}^{S_{2}}\right)^{T}\left(P_{j}^{S_{2}}\right)^{-T} \hat{x}_{j}^{S_{2}}\right) \\
\zeta_{i j} & =-\frac{1}{2}\left(d \log 2 \pi-\log \left|P_{i j}^{-1}\right|+\hat{x}_{i j}^{T} P_{i j}^{-T} \hat{x}_{i j}\right)
\end{aligned}
$$

The derivations of Eqs. (16) and (17) are presented in Appendix.

Given the fusion rule, the next issue to investigate is the determination of the weighting coefficient $w$. Here we first define a cost function $J(w)$, then the problem becomes to find the optimal $w^{*}$ so that

$$
w^{*}=\arg \min _{w \in[0,1]} J(w)
$$

One possible criterion is to minimize the trace of the fused estimation covariance, which can be interpreted as minimization of the Shannon information of $p^{f}(x)$ [13]. However, when the fused result follows Gaussian mixture distribution, the covariance is no longer a sufficiently good description of estimation uncertainty. An alternative solution is to adopt Kullback-Leibler divergence (KLD [30]) as a measure of the discrepancy between two random variables. Hurley [28] proposed that when the KLD of $p^{f}(x)$ from $p^{S_{1}}(x)$ and $p^{S_{2}}(x)$ is identical, the resulting fused result represents the minimization of Chernoff information. In this regard, Eq. (18) can be formulated as

$$
J(w)=\left(D\left(p^{f} \| p^{S_{1}}\right)-D\left(p^{f} \| p^{S_{2}}\right)\right)^{2}
$$

where the $\operatorname{KLD} D(\cdot \| \cdot)$ is expressed as

$$
D(p \| q)=\int p(x) \log \frac{p(x)}{q(x)} d x
$$

Although the KLD between two Gaussian mixture distributions is not analytically achievable, some techniques have been developed to overcome this problem by replacing the KLD with other approximation functions which can be calculated efficiently. In this study, we use the variational method [31] to approximate the KLD, then the cost function $J(w)$ in Eq. (19) is calculated with $w$ varying, finally the optimal $w^{*}$ is determined with exhaustive search over the grid in $[0,1]$ specified by the increment $\Delta$.

In summary, the proposed approximate Chernoff fusion algorithm for Gaussian mixtures is presented in Algorithm 1.

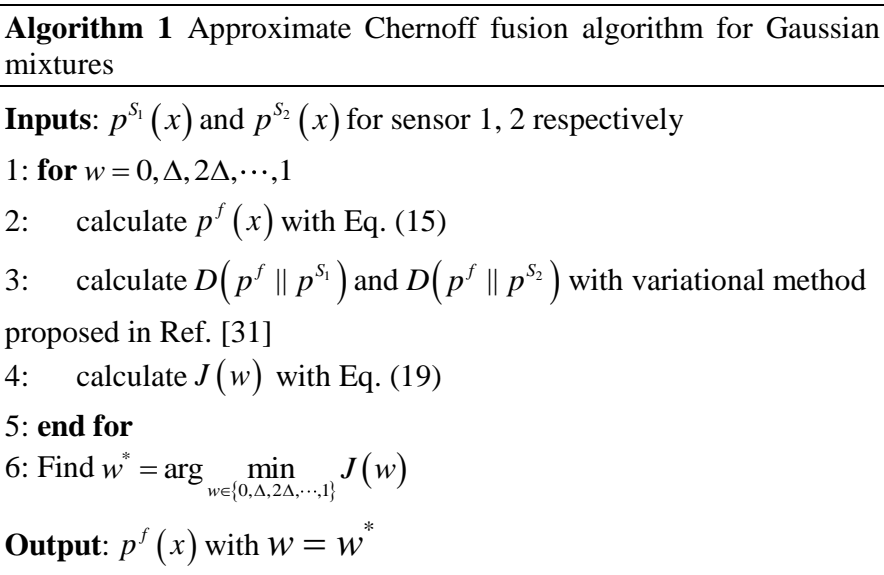

\section{Simulation results}

In this section, simulations have been carried out to verify the effectiveness of the proposed fusion method. For the target dynamics, we consider a system in Eq. (1) with $g=9.81 \mathrm{~m} \cdot \mathrm{s}^{-2}, T=2 s$ and $q=1 \mathrm{~m}^{2} \cdot \mathrm{s}^{-3}$. Besides, the initial state $x_{0}$ is given as

$$
x_{0}=\left[\begin{array}{c}
23200 \mathrm{~m} \\
2290 \cos \left(190^{\circ}\right) \mathrm{m} \cdot \mathrm{s}^{-1} \\
88000 \mathrm{~m} \\
2290 \sin \left(190^{\circ}\right) \mathrm{m} \cdot \mathrm{s}^{-1}
\end{array}\right]
$$

and the initial filtering covariance is

$$
P_{0}=\operatorname{diag}\left(1000^{2} \mathrm{~m}^{2}, 20^{2} \mathrm{~m}^{2} \cdot \mathrm{s}^{-2}, 1000^{2} \mathrm{~m}^{2}, 20^{2} \mathrm{~m}^{2} \cdot \mathrm{s}^{-2}\right)
$$

The air density $\rho(\cdot)$ is modelled piecewise as

$$
\rho\left(y_{p}\right)=\left\{\begin{array}{l}
1.227 e^{-1.093 y_{p} \times 10^{-4}}, y_{p}<9144 \mathrm{~m} \\
1.754 e^{-1.49 y_{p} \times 10^{-4}}, y_{p} \geq 9144 \mathrm{~m}
\end{array}\right.
$$


And he actual target ballistic coefficient is set to be $\beta=4 \times 10^{4} \mathrm{~kg} \cdot \mathrm{m}^{-1} \cdot \mathrm{s}^{-2}$.

Fig. 4 plots the ballistic target trajectory in the X$Y$ plane. Fig. 5 shows the velocity of target against time, Fig. 6 depicts the aerodynamic drag acceleration versus time. By comparing Figs. 5 and 6 , one can observe the nonlinear deceleration of the target due to the aerodynamic drag.

For the measurement model given in Eq. (9), the corresponding simulation parameters are configured as follows: $R_{r}^{S_{1}}=R_{r}^{S_{1}}=100^{2} \mathrm{~m}^{2}$, $R_{\theta}^{S_{1}}=R_{\theta}^{S_{2}}=0.05^{2} \mathrm{rad}^{2}$. Besides, we consider that the sensors are located at $\left(x_{R}^{S_{1}}, y_{R}^{S_{1}}\right)=(0,0)$ and $\left(x_{R}^{S_{2}}, y_{R}^{S_{2}}\right)=\left(1.5 \times 10^{5} \mathrm{~m}, 0\right)$ respectively.

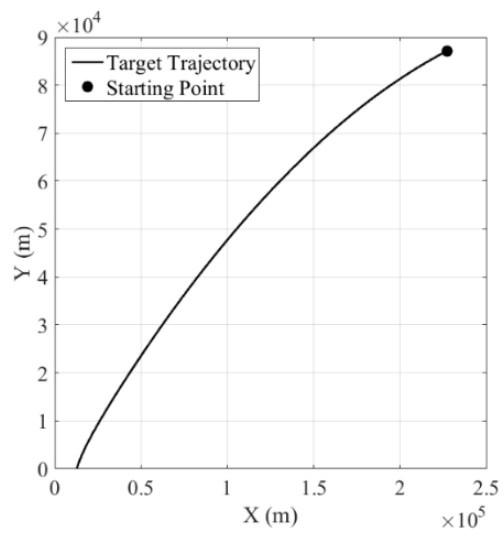

Fig. 4 Ballistic target trajectory

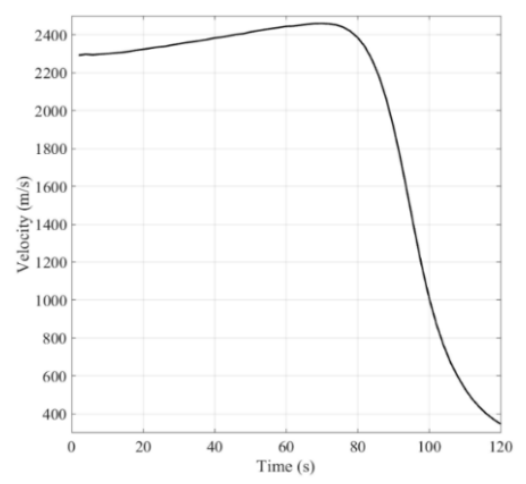

Fig. 5 Velocity of ballistic target against time

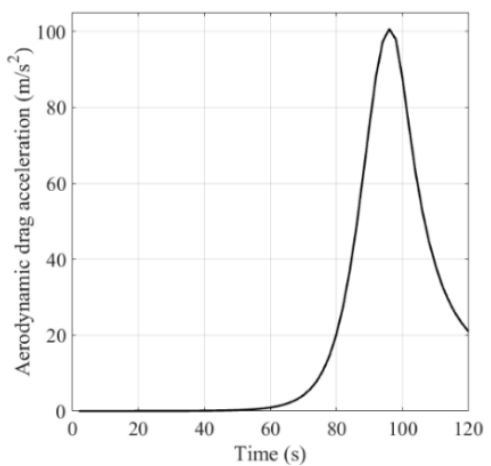

Fig. 6. Aerodynamic drag acceleration against time
We first evaluate the performance of local IMM tracker at the single sensor level. A total of five different model configurations are designed for comparison purposes, in which the local IMM filter might use two or three models, and the corresponding model parameters $\beta_{i}$ are given as follows:

1) CFG-1: A total of 3 filter models, $\beta_{1}=1 \times 10^{4}$, $\beta_{2}=3 \times 10^{4}, \beta_{3}=5 \times 10^{4}$;

2) CFG-2: A total of 3 filter models, $\beta_{1}=3 \times 10^{4}$, $\beta_{2}=5 \times 10^{4}, \beta_{3}=7 \times 10^{4}$;

3) CFG-3: A total of 2 filter models, $\beta_{1}=1 \times 10^{4}$, $\beta_{2}=7 \times 10^{4}$;

4) CFG-4: A total of 2 filter models, $\beta_{1}=2 \times 10^{4}$, $\beta_{2}=6 \times 10^{4}$;

5) CFG-5: A total of 2 filter models, $\beta_{1}=3 \times 10^{4}$, $\beta_{2}=5 \times 10^{4}$.

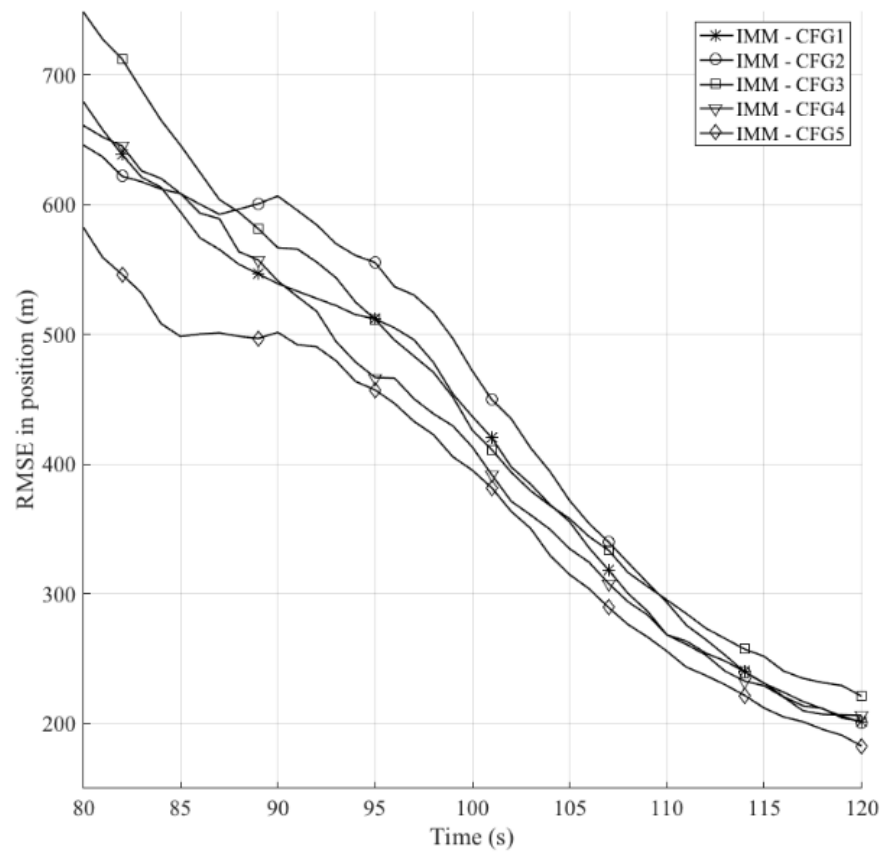

Fig. 7 Comparison of RMSE for IMM filter under different model configurations

Fig. 7 compares the performance of IMM filter in terms of root mean squared error (RMSE) in position under five different model configurations. Fig. 8 shows the corresponding averaged RMSE over the last twenty steps (from 100s to 120s). It can be observed that the local tracker gets the best result with only two models under configuration 5 , which suggests that the estimation accuracy of IMM filter cannot be improved by simply increasing the 
number of filter models, in fact the accuracy of filter models matters more than the size of the filter model set. Unfortunately, it is not realistic to predetermine an optimal model set before IMM tracking in practice, because that the exact model parameter (like the ballistic coefficient $\beta$ ) is usually not available to the local trackers. Furthermore, when we consider a distributed target tracking framework in which local sensors use IMM filter, it is better to set the filters to follow different model configurations in the sense of robustness.

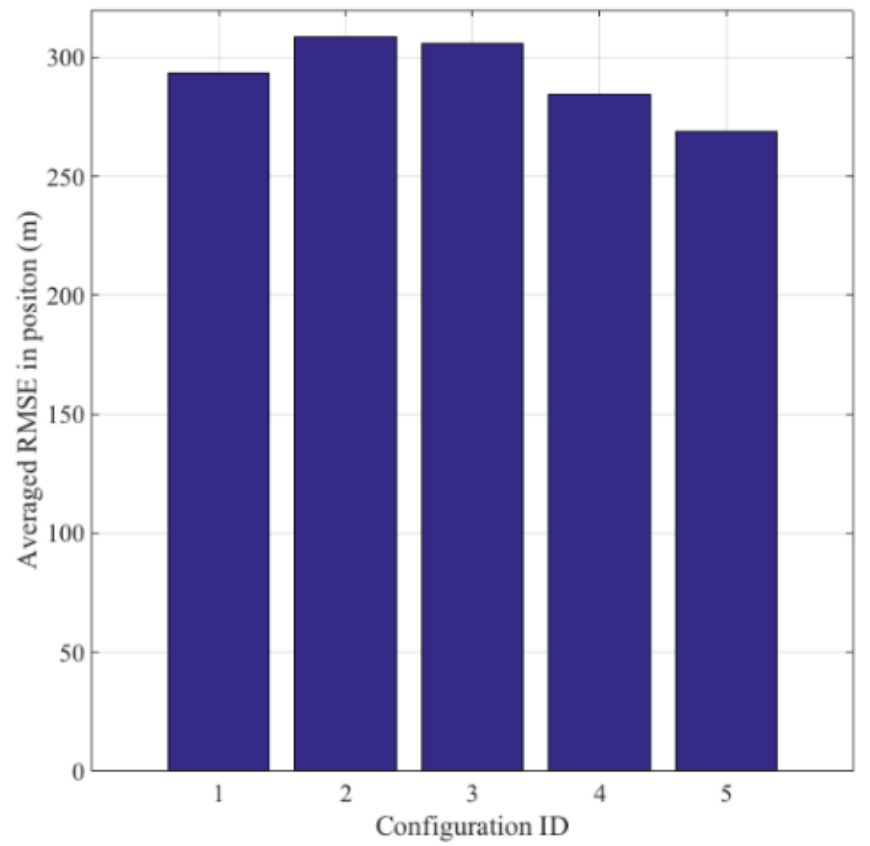

Fig. 8 Comparison of Averaged RMSE for IMM filter under different configurations

Secondly, we compare the proposed approximate Chernoff fusion (ACF) method with several popular fusion methods available in open literature, including simple convex combination (CC) method [32], covariance intersection (CI) method [18], ellipse intersection (EI) method [33] and a Gaussian mixtures fusion method based on approximate Bayesian fusion (ABF) [34, 35]. Note that CC, CI and EI only applies to the Gaussian case, as a result they require to extract an approximate version of means and covariances from the IMM outputs with moment matching rule [36] to fuse. The development of $\mathrm{ABF}$ is based on Eq. (11). Instead of pre-processing the IMM outputs, $A B F$ approximates the common prior information with a single Gaussian PDF to derive a fused result with a closed form.

In the simulation, we consider sensors $S_{1}$ and $S_{2}$ run local IMM filter under configuration 2 and 3 respectively, while both two configurations are not best choices at the single sensor level, we choose them with aims to verify the effectiveness of the proposed fusion algorithm under adverse environment.

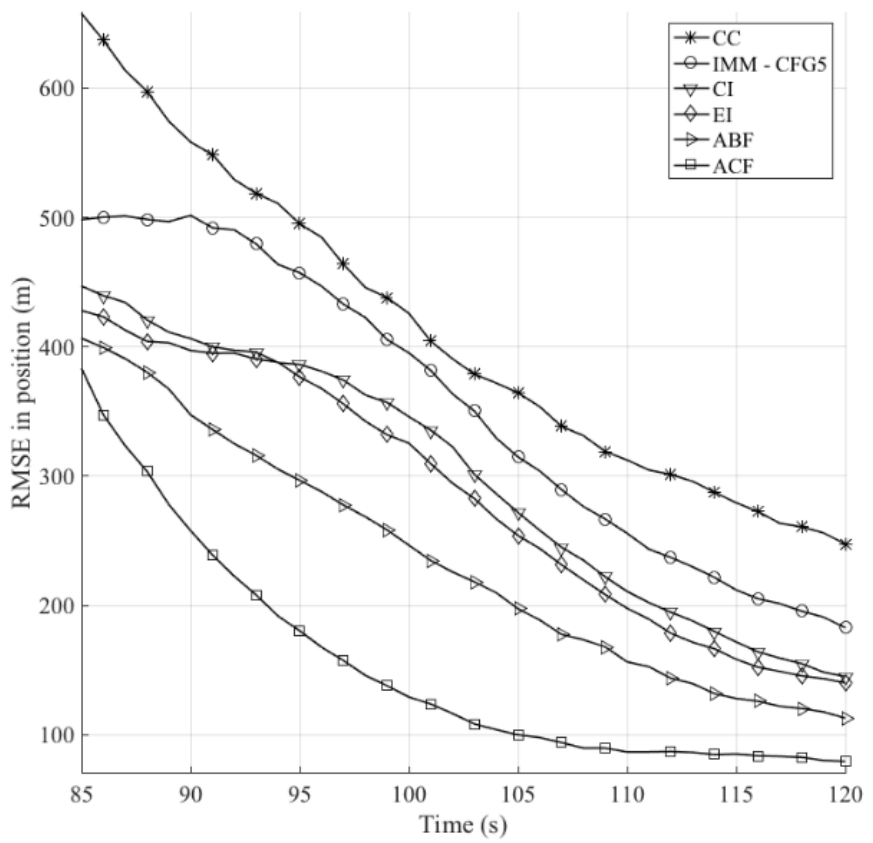

Fig. 9 Comparison of RMSE between different fusion algorithms and IMM-CFG5

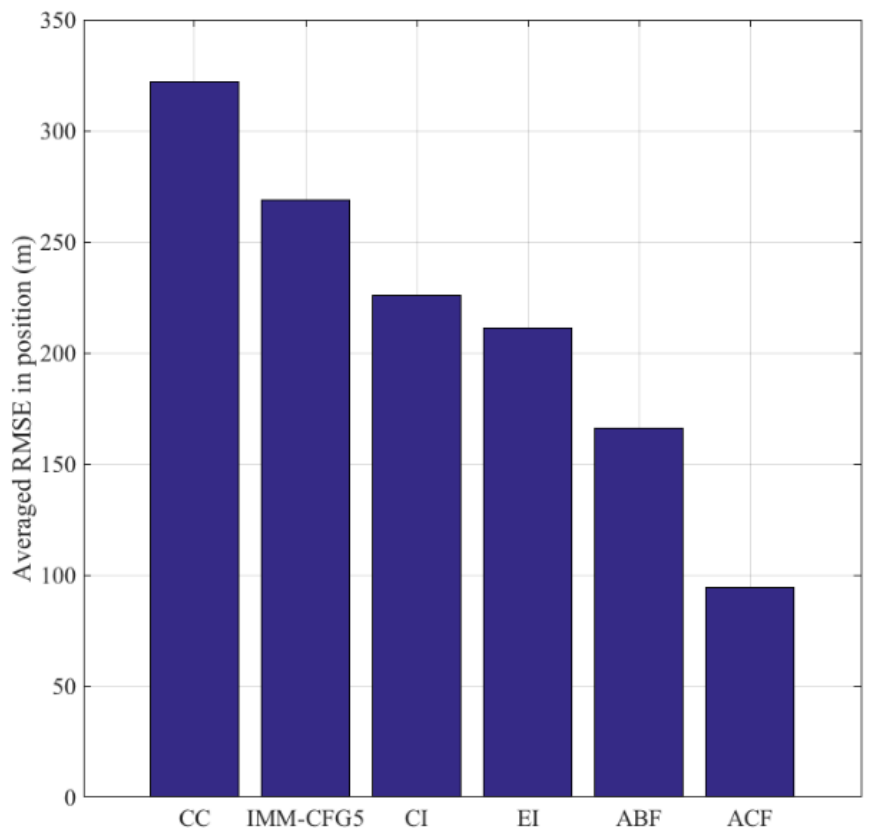

Fig. 10 Comparison of Averaged RMSE between different fusion algorithms and IMM-CFG5

Figs. 9 and 10 compare the performance of different fusion algorithms in the sense of RMSE and averaged RMSE respectively. First, the results from $\mathrm{CI}, \mathrm{EI}, \mathrm{ABF}$ and $\mathrm{ACF}$ are all better than that from the best single sensor case (IMM-CFG5), which suggests that one could improve the 
estimation accuracy beyond the single sensor level by using these sensor fusion techniques. The CC method performs even worse than IMM-CFG5, this result proves that the correlation between the local estimates cannot be simply ignored during the fusion process. Secondly, it can be observed that the Gaussian mixtures-based fusion method (ABF and the proposed $\mathrm{ACF}$ ), both perform better than the traditional Gaussian-based fusion method (CI and $\mathrm{EI})$. The reason is that when we directly fuse local estimates in terms of Gaussian mixtures, it enables us to sufficiently exploit their original information about the probability distribution. However, for CI and EI, the information loss is inevitable during the conversion from IMM outputs to their momentmatching approximations. Furthermore, it appears that the proposed ACF is significantly better than $\mathrm{ABF}$. It's because ABF assumes that the common information can be modelled as a single Gaussian $\mathrm{PDF}$, while in practice the redundant information to be removed follow Gaussian mixture distribution, therefore the fusion performance of $\mathrm{ABF}$ degrades due to its imprecise assumption.

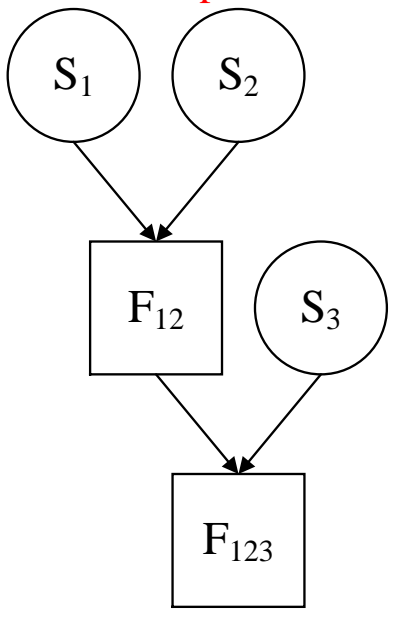

Fig. 11 Hierarchical fusion structure with three sensors

In the end, we extend the two-sensor T2TF architecture shown in Fig. 1 to make it incorporate more sensors. As shown in Fig. 11, here we introduce a hierarchical fusion architecture which can be used to fuse multiple tracks in a sequential manner. Suppose that there are three sensors with their local estimates indexed as $S_{i}, i=1,2,3$, then we first fuse estimates from the first two sensors, i.e., $S_{1}$ and $S_{2}$ to generate a fused track $F_{12}$, after that we fuse $F_{12}$ with the local estimate $S_{3}$ from the third sensor. Consequently, this fusion architecture is scalable as it can handle arbitrary number of sensors.
In the following, we conduct simulations to analyze its performance improvement with varying number of sensors. For the sake of brevity, we consider that all the local sensors are homogeneous, and all of them use local IMM filter under configuration 5. Figs. 12 and 13 compare the performance of the ACF with different number of local sensors. The results show that the global estimation accuracy can be improved by fusing more local tracks under this hierarchical fusion architecture with our proposed $\mathrm{ACF}$ algorithm. 


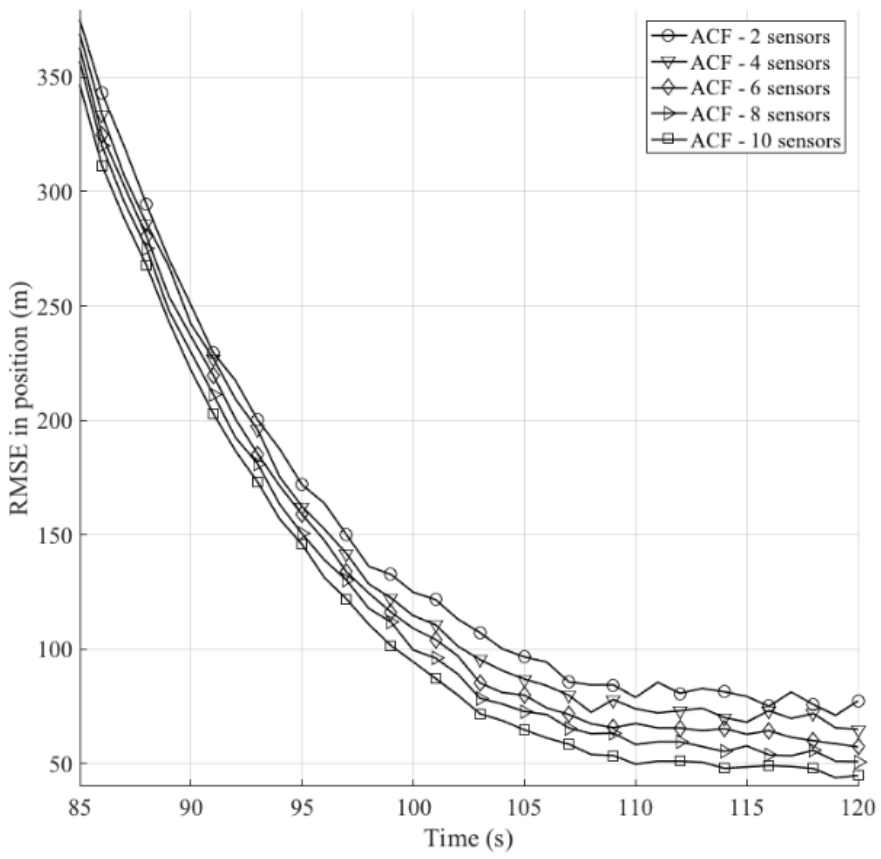

Fig. 12 Comparison of RMSE with different number of sensors for $\mathrm{ACF}$

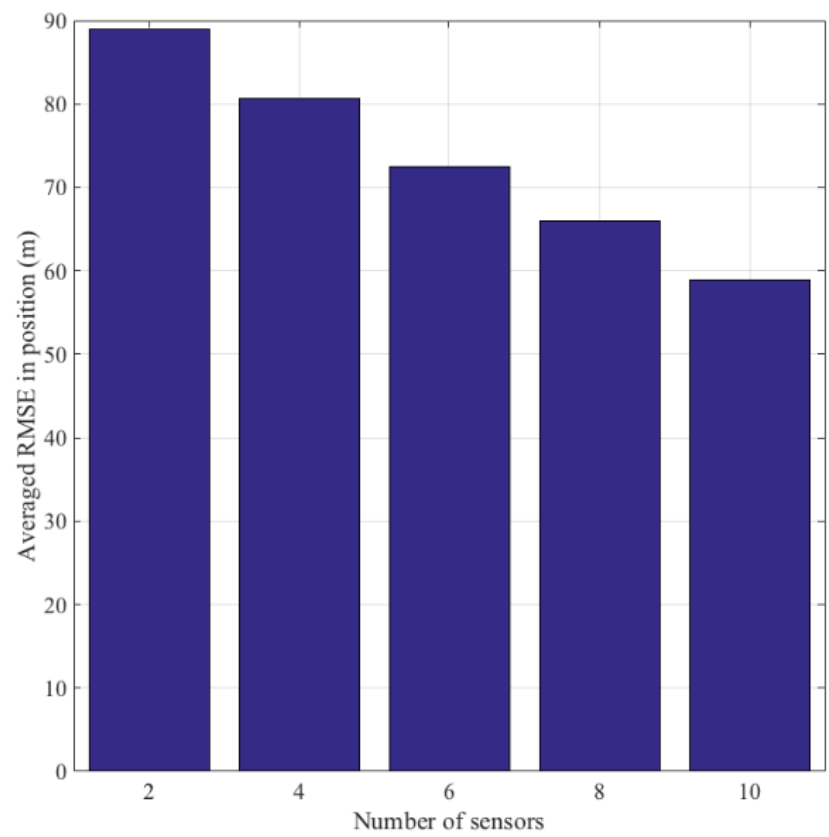

Fig. 13 Comparison of averaged RMSE with different number of sensors for ACF

\section{Conclusion}

In this study, an approximate Chernoff fusion method for Gaussian mixture model is presented. An arbitrary power of a Gaussian mixture distribution is approximated with Gaussian mixture model using first order expansion approximation. It shows that such approximation can lead to an analytical fusion equation for Gaussian mixtures.
The developed fusion methodology is verified by tracking a re-entry ballistic vehicle with sensor networks. Monte Carlo simulations have been conducted to prove the effectiveness of the proposed fusion method.

\section{Acknowledgements}

This work was partially supported by the National Science Foundation of China (Grant No. 61273349 and 61573042).

\section{Appendix}

A multivariate Gaussian $\operatorname{PDF} N\left(x ; \hat{x}_{i}, P_{i}\right)$ can be written in canonical notation as

$$
N\left(x ; \hat{x}_{i}, P_{i}\right) \square \exp \left(\zeta_{i}+y_{i}^{T} x-\frac{1}{2} x^{T} Y_{i} x\right)
$$

where

$$
\begin{aligned}
Y_{i} & =P_{i}^{-1} \\
y_{i} & =P_{i}^{-1} \hat{x}_{i} \\
\zeta_{i} & =-\frac{1}{2}\left(d \log 2 \pi-\log \left|Y_{i}\right|+y_{i}^{T} Y_{i}^{-1} y_{i}\right)
\end{aligned}
$$

and $d$ is the dimensionality of $x \cdot\left|Y_{i}\right| \square \operatorname{det}\left(Y_{i}\right)$. Then we have

$$
\begin{aligned}
& N\left(x ; \hat{x}_{i}, P_{i}\right) \cdot N\left(x ; \hat{x}_{j}, P_{j}\right) \\
= & \exp \left(\zeta_{i}+y_{i}^{T} x-\frac{1}{2} x^{T} Y_{i} x\right) \\
& \cdot \exp \left(\zeta_{j}+y_{j}^{T} x-\frac{1}{2} x^{T} Y_{j} x\right) \\
= & \exp \left(\left(\zeta_{i}+\zeta_{j}\right)+\left(y_{i}+y_{j}\right)^{T} x\right. \\
& \left.-\frac{1}{2} x^{T}\left(Y_{i}+Y_{j}\right) x\right)
\end{aligned}
$$

We further define

$$
\begin{aligned}
& Y_{i j} \square Y_{i}+Y_{j} \\
& y_{i j} \square y_{i}+y_{j} \\
& \zeta_{i j} \square-\frac{1}{2}\left(d \log 2 \pi-\log \left|Y_{i j}\right|+y_{i j}^{T} Y_{i j}^{-1} y_{i j}\right)
\end{aligned}
$$

Then Eq. (23) can be rewritten as 


$$
\begin{aligned}
& N\left(x ; \hat{x}_{i}, P_{i}\right) \cdot N\left(x ; \hat{x}_{j}, P_{j}\right) \\
= & \exp \left(\left(\zeta_{i}+\zeta_{j}\right)-\zeta_{i j}+\zeta_{i j}+y_{i j}^{T} x-\frac{1}{2} x^{T} Y_{i j} x\right) \\
= & \exp \left(\zeta_{i}+\zeta_{j}-\zeta_{i j}\right) \cdot \exp \left(\zeta_{i j}+y_{i j}^{T} x-\frac{1}{2} x^{T} Y_{i j} x\right)
\end{aligned}
$$

Eq. (25) shows that the product of two Gaussian PDFs is a scaled Gaussian PDF as

$$
N\left(x ; \hat{x}_{i}, P_{i}\right) \cdot N\left(x ; \hat{x}_{j}, P_{j}\right)=c_{i j} N\left(x ; \hat{x}_{i j}, P_{i j}\right)
$$

where

$$
\begin{gathered}
P_{i j}^{-1}=P_{i}^{-1}+P_{j}^{-1} \\
P_{i j}^{-1} \hat{x}_{i j}=P_{i}^{-1} \hat{x}_{i}+P_{j}^{-1} \hat{x}_{j}
\end{gathered}
$$

and the scaling factor is

$$
c_{i j}=\exp \left(\zeta_{i}+\zeta_{j}-\zeta_{i j}\right)
$$

with

$$
\begin{aligned}
& \zeta_{i}=-\frac{1}{2}\left(d \log 2 \pi-\log \left|P_{i}^{-1}\right|+\hat{x}_{i}^{T} P_{i}^{-T} \hat{x}_{i}\right) \\
& \zeta_{j}=-\frac{1}{2}\left(d \log 2 \pi-\log \left|P_{j}^{-1}\right|+\hat{x}_{j}^{T} P_{j}^{-T} \hat{x}_{j}\right) \\
& \zeta_{i j}=-\frac{1}{2}\left(d \log 2 \pi-\log \left|P_{i j}^{-1}\right|+\hat{x}_{i j}^{T} P_{i j}^{-T} \hat{x}_{i j}\right)
\end{aligned}
$$

In this regard, for the product of $N\left(x ; \hat{x}_{i}^{S_{1}}, \frac{P_{i}^{S_{1}}}{w}\right)$ and $N\left(x ; \hat{x}_{j}^{S_{2}}, \frac{P_{j}^{S_{2}}}{1-w}\right)$, we have

$$
\begin{aligned}
& N\left(x ; \hat{x}_{i}^{S_{1}}, \frac{P_{i}^{S_{1}}}{w}\right) \cdot N\left(x ; \hat{x}_{j}^{S_{2}}, \frac{P_{j}^{S_{2}}}{1-w}\right) \\
& =c_{i j} N\left(x ; \hat{x}_{i j}, P_{i j}\right)
\end{aligned}
$$

where

$$
\begin{aligned}
P_{i j}^{-1} & =w\left(P_{i}^{S_{1}}\right)^{-1}+(1-w)\left(P_{j}^{S_{2}}\right)^{-1} \\
P_{i j}^{-1} \hat{x}_{i j} & =w\left(P_{i}^{S_{1}}\right)^{-1} \hat{x}_{i}^{S_{1}}+(1-w)\left(P_{j}^{S_{2}}\right)^{-1} \hat{x}_{j}^{S_{2}}
\end{aligned}
$$

and

$$
c_{i j}=\exp \left(\zeta_{i}^{S_{1}}+\zeta_{j}^{S_{2}}-\zeta_{i j}\right)
$$

with

$$
\begin{aligned}
\zeta_{i}^{S_{1}} & =-\frac{1}{2}\left(d \log 2 \pi-\log \left|w\left(P_{i}^{S_{1}}\right)^{-1}\right|\right. \\
& \left.+w\left(\hat{x}_{i}^{S_{1}}\right)^{T}\left(P_{i}^{S_{1}}\right)^{-T} \hat{x}_{i}^{S_{1}}\right) \\
\zeta_{j}^{S_{2}} & =-\frac{1}{2}\left(d \log 2 \pi-\log \left|(1-w)\left(P_{j}^{S_{2}}\right)^{-1}\right|\right. \\
& \left.+(1-w)\left(\hat{x}_{j}^{S_{2}}\right)^{T}\left(P_{j}^{S_{2}}\right)^{-T} \hat{x}_{j}^{S_{2}}\right) \\
\zeta_{i j} & =-\frac{1}{2}\left(d \log 2 \pi-\log \left|P_{i j}^{-1}\right|+\hat{x}_{i j}^{T} P_{i j}^{-T} \hat{x}_{i j}\right)
\end{aligned}
$$

\section{References}

[1] T. Yuan, Y. Bar-Shalom, P. Willett, and E. Mozeson, "A Multiple IMM Estimation Approach with Unbiased Mixing for Thrusting Projectiles," IEEE Transactions on Aerospace \& Electronic Systems, vol. 48, pp. 3250-3267, 2012.

[2] S. Bhaumik, N. K. Singh, and S. Bhattacharya, "A comparison of several nonlinear filters for ballistic missile tracking on re-entry," in 2016 IEEE First International Conference On Control, Measurement And Instrumentation (CMI), 2016.

[3] A. Benavoli, L. Chisci, and A. Farina, "Tracking of a Ballistic Missile with APriori Information," IEEE Transactions on Aerospace \& Electronic Systems, vol. 43, pp. 1000-1016, 2007.

[4] A. Farina, B. Ristic, and D. Benvenuti, "Tracking a ballistic target: comparison of several nonlinear filters," IEEE Transactions on Aerospace and Electronic Systems, vol. 38, pp. 854-867, 2002.

[5] X. R. Li and V. P. Jilkov, "Survey of Maneuvering Target Tracking. Part II: Motion Models of Ballistic and Space Targets," IEEE Transactions on Aerospace \& Electronic Systems, vol. 46, pp. 96-119, 2010.

[6] N. Gageik, P. Benz, and S. Montenegro, "Obstacle Detection and Collision Avoidance for a UAV With Complementary Low-Cost Sensors," Access IEEE, vol. 3, pp. 1-1, 2015.

[7] M. S. Challa, J. G. Moore, and D. J. Rogers, "A Simple Attitude Unscented Kalman 
Filter: Theory and Evaluation in a Magnetometer-Only Spacecraft Scenario," IEEE Access, vol. 4, pp. 1845-1858, 2016.

[8] J. D. Park and J. F. Doherty, "Track Detection of Low Observable Targets Using a Motion Model," Access IEEE, vol. 3, pp. 1408-1415, 2015.

[9] F. Reali, G. Palmerini, A. Farina, A. Graziano, and L. Timmoneri, "Tracking a ballistic target by multiple model approach," in Aerospace conference, 2009 IEEE, 2009, pp. 1 - 14.

[10] G. Battistelli, L. Chisci, C. Fantacci, and A. Farina, "Consensus-based multiple-model Bayesian filtering for distributed tracking," Iet Radar Sonar Navigation, vol. 9, pp. 401410, 2015.

[11] W. Zhu, W. Wang, and G. Yuan, "An Improved Interacting Multiple Model Filtering Algorithm Based on the Cubature Kalman Filter for Maneuvering Target Tracking," Sensors, vol. 16, p. 805, 2016.

[12] B. N. Vo and W. K. Ma, "The Gaussian Mixture Probability Hypothesis Density Filter," IEEE Transactions on Signal Processing, vol. 54, pp. 4091-4104, 2010.

[13] K. Chang, C.-Y. Chong, and S. Mori, "Analytical and computational evaluation of scalable distributed fusion algorithms," Aerospace and Electronic Systems, IEEE Transactions on, vol. 46, pp. 2022-2034, 2010.

[14] K. C. Chang, T. Zhi, and S. Mori, "Performance evaluation for MAP state estimate fusion," IEEE Transactions on Aerospace and Electronic Systems, vol. 40, pp. 706-714, 2004.

[15] J. Roecker and D. Theisen, "Multiple sensor tracking architecture comparison," Aerospace and Electronic Systems Magazine, IEEE, vol. 29, pp. 28-33, 2014.

[16] S. Grime and H. Durrant-Whyte, "Communication in decentralized systems," Ifac Control Engineering Practice, 1994.

[17] T. W. Martin and K.-C. Chang, "A distributed data fusion approach for mobile ad hoc networks," in Information Fusion, 2005 8th International Conference on, 2005, p. 8 pp.
[18] S. J. Julier and J. K. Uhlmann, "General decentralized data fusion with covariance intersection (CI)," 2001.

[19] X. Tian and Y. Bar-Shalom, "Exact algorithms for four track-to-track fusion configurations: All you wanted to know but were afraid to ask," in Information Fusion, 2009. FUSION'09. 12th International Conference on, 2009, pp. 537-544.

[20] A. Farina, D. Benvenuti, and B. Ristic, "Estimation accuracy of a landing point of a ballistic target," in Information Fusion, 2002. Proceedings of the Fifth International Conference on, 2002, pp. 2-9 vol.1.

[21] Z. Zhao, H. Chen, G. Chen, C. Kwan, and X. R. Li, "Comparison Of Several Ballistic Target Tracking Filters," in American Control Conference, 2006.

[22] S.-J. Shin, "Re-entry vehicle tracking with a new multiple model estimation applicable to highly non-linear dynamics," Radar, Sonar \& Navigation, IET, vol. 9, pp. 581-588, 2015.

[23] W. Farrell, "Interacting multiple model filter for tactical ballistic missile tracking," IEEE Transactions on Aerospace \& Electronic Systems, vol. 44, pp. 418-426, 2008.

[24] Y. Bar-Shalom, X. R. Li, and T. Kirubarajan, Estimation with Applications to Tracking and Navigation, Theory, Algorithms, and Software: Wiley-Inter-Science, 2008.

[25] C.-Y. Chong, S. Mori, W. H. Barker, and K.-C. Chang, "Architectures and algorithms for track association and fusion," Aerospace and Electronic Systems Magazine, IEEE, vol. 15, pp. 5-13, 2000.

[26] K. Chang, C.-Y. Chong, and S. Mori, "On scalable distributed sensor fusion," in Information Fusion, 2008 11th International Conference on, 2008, pp. 1-8.

[27] D. Hall, C. Y. Chong, J. Llinas, and M. Liggins, "Distributed Data Fusion for Network-Centric Operations," CRC Press, 2012.

[28] M. B. Hurley, "An information theoretic justification for covariance intersection and its generalization," in Information Fusion, 2002. Proceedings of the Fifth International Conference on, 2002, pp. 505-511. 
[29] A. R. Runnalls, "Kullback-Leibler Approach to Gaussian Mixture Reduction," IEEE Transactions on Aerospace \& Electronic Systems, vol. 43, pp. 989-999, 2007.

[30] S. Kullback and R. A. Leibler, "On information and sufficiency," The Annals of Mathematical Statistics, pp. 79-86, 1951.

[31] J. R. Hershey and P. A. Olsen, "Approximating the Kullback Leibler Divergence Between Gaussian Mixture Models," in IEEE International Conference on Acoustics, 2007, pp. IV-317 - IV-320.

[32] C.-Y. Chong and S. Mori, "Convex combination and covariance intersection algorithms in distributed fusion," in 4th Int. Conf. Information Fusion, Montreal, Canada, 2001.
[33] J. Sijs and M. Lazar, "State fusion with unknown correlation: Ellipsoidal intersection," Automatica, vol. 48, pp. 18741878, 2012.

[34] K.-C. Chang and W. Sun, "Scalable fusion with mixture distributions in sensor networks," in Control Automation Robotics \& Vision (ICARCV), 2010 11th International Conference on, 2010, pp. 1251-1256.

[35] K. Lu and Z. Rui, "Sensor Fusion of Gaussian Mixtures for Ballistic Target Tracking in the Re-Entry Phase," Sensors, vol. 16, 2016.

[36] Y. Bar-Shalom and X.-R. Li, "Estimation with Applications to Tracking and Navigation," 2001. 